\title{
Calidad del agua de uso doméstico en la parroquia Alonso de Ojeda del estado Zulia-Venezuela
}

\author{
Quality of water for domestic use in the Alonso de Ojeda parish of the \\ Zulia state-Venezuela
}

\author{
Lorena Fuentes Spooner \\ Ifuentesp89@gmail.com \\ ORCID 0000-0002-9037-4692 \\ Universidad del Zulia, Venezuela
}

\author{
José Colina Barboza \\ colina66@hotmail.com \\ ORCID 0000-0001-5251-5370 \\ Universidad del Zulia, Venezuela
}

Artículo recibido mayo 2019 | Arbitrado en julio 2019 | Publicado en septiembre 2019

\section{RESUMEN}

El consumo de agua que no se ajusta a los estándares de calidad establecidos, implica riesgos para la salud de la población. El objetivo de esta investigación fue determinar la calidad del agua de uso doméstico en la parroquia Alonso de Ojeda, municipio Lagunillas del estado Zulia, Venezuela. Se desarrolló una investigación descriptiva y la muestra estuvo representada por el agua de grifo de cien viviendas de la parroquia, la cual se caracterizó fisicoquímicamente y microbiológicamente. Los resultados arrojaron que las muestras no cumplieron con las normas venezolanas en cuanto a turbidez $(22,86$ UNT), color $(39,60 \mathrm{UC}$ Pt-Co) y coliformes totales ( $\geq 16 \mathrm{NMP}$ ). El pH se ajustó a la normativa venezolana $(\mathrm{pH}=6,55)$ y la alcalinidad se ubicó en 14,98 mg CaCO3/L, aceptable según la normativa colombiana. Se concluye que el agua suministrada a la población de la parroquia Alonso de Ojeda no es apta para el consumo humano.

Palabras clave: Agua de uso doméstico, calidad del agua, coliformes totales, parámetros fisicoquímicos

\begin{abstract}
The consumption of water that does not comply with the established quality standards implies health risks for the population. The objective of this investigation was to determine the quality of water for domestic use in the Alonso de Ojeda parish, Lagunillas municipality of the Zulia state, Venezuela. A descriptive investigation was carried out and the sample was represented by tap water from one hundred houses in the parish, which was characterized physicochemically and microbiologically. The results showed that the samples did not meet the Venezuelan standards regarding turbidity (22.86 UNT), color (39.60 UC Pt-Co) and total coliforms ( $\geq 16$ NMP). The $\mathrm{pH}$ was adjusted to Venezuelan regulations $(\mathrm{pH}=6.55)$ and the alkalinity was $14.98 \mathrm{mg} \mathrm{CaCO} 3$ / L, acceptable according to Colombian regulations. It is concluded that the water supplied to the population of the Alonso de Ojeda parish is not suitable for human consumption.
\end{abstract}

Key words: Water for domestic use, water quality, total coliforms, physicochemical parameters 
INTRODUCCIÓN

La importancia del agua para la existencia de la vida en la Tierra es una verdad innegable, sus usos y aplicaciones abarcan desde simples tareas domésticas hasta labores industriales, su utilización para el riego y cultivo en las zonas agrícolas es indispensable $\mathrm{y}$ necesaria en grandes cantidades.

Según datos estadísticos, más de 1,5 millones de niños mueren anualmente a causa de las enfermedades que se transmiten por el agua contaminada. Y por si fuera poco aproximadamente 1.100 millones de personas carecen de acceso a una fuente mejorada de abastecimiento de agua. Alrededor de 2.500 millones de personas en el mundo viven sin un sistema adecuado de saneamiento y 884 millones de personas, la mayoría de ellas africanas, no tienen acceso al agua potable. Cada día 2 millones de toneladas de aguas residuales desembocan en las aguas del mundo. En los países en desarrollo, más del $90 \%$ de los desechos sin procesar y el $70 \%$ de los desechos industriales sin tratar se vierten en aguas superficiales. Los recursos hídricos están cada vez más amenazados por la contaminación de los últimos 50 años. En las zonas costeras, ecosistemas como los manglares, los lechos de algas y los arrecifes de coral están desapareciendo a una velocidad alarmante a causa de la contaminación del agua (UNESCO, 2011).

La disponibilidad de agua en cantidad y calidad es esencial para el desarrollo económico y social de los continentes, los países y las regiones. En América Latina hay grandes ríos como el Amazonas, el Orinoco o el Magdalena, pero también reservas menos conocidas como el Acuífero Guaraní, que alberga más de $40.000 \mathrm{~km}^{3}$ de agua debajo de Argentina, Paraguay, Uruguay y Brasil. Si a esto se suman las nieves eternas de las cordilleras y los glaciares del Cono Sur, América Latina dispone del $65 \%$ del agua dulce del mundo, según cálculos del Programa Ambiental de las Naciones Unidas (UNEP, 2010).

El agua destinada al consumo humano debe cumplir con una serie de características fisicoquímicas y microbiológicas que permitan asegurar su calidad y garantizar que es potable. Algunos estudios evidencian el cumplimiento de los estándares establecidos, pero otros no se ajustan a las normativas establecidas. Particularmente, en Colombia se han reportado investigaciones que han logrado determinar el cumplimiento de todos los parámetros de potabilidad exigidos de acuerdo con la resolución 2115/2007 para agua destinada al consumo humano (Fajardo, Muñoz-Silva, Otero y Mendoza, 2017); no obstante, otras no se ajustan a dicha resolución, como por ejemplo, la realizada en el municipio Bojacá, Cundinamarca, la cual reflejó que las muestras de agua potable analizadas cumplieron con lo establecido en cuanto a parámetros como pH, turbidez, hierro, alcalinidad, dureza y aluminio, pero el cloro residual no se ajustó a los valores permisibles establecidos, de modo que el agua no resultó apta para el consumo humano (Estupiñán y Ávila, 2010); y la efectuada en el departamento del Tolima, donde el 63,83 \% de los municipios presentaron agua no potable y el $27,7 \%$ de los municipios evidenciaron resultados con coliformes (Bríñez, Guarnizo y Arias, 2012).

En Ecuador también se han reportado resultados similares, como el de un estudio realizado en el cantón Quevedo, provincia de Los Ríos, el cual arrojó que los parámetros: nitritos, nitratos, turbidez, sólidos disueltos totales, $\mathrm{pH}$, dureza total, color y hierro, se encontraron en el rango de aceptabilidad de calidad ambiental; pero, los valores 
manganeso y oxígeno disuelto sobrepasaron los límites máximos permisibles por el Texto Unificado de Legislación Secundaria del Medio Ambiente (TULSMA), al igual que los coliformes totales y fecales (Baque-Mite et al., 2016).

Específicamente, se conoce que Venezuela cuenta con abundantes recursos hídricos distribuidos en siete sistemas hidrográficos y 16 regiones hidrográficas, especialmente al sur de este país (Rodríguez-Betancourt y González-Aguirre, 2000). Se ha calculado que el volumen de los recursos hídricos es de $1.320 \mathrm{~km}^{3}$ por año (Cañizales et al., 2006 citado por Jiménez y Galizia, 2012). El proceso de ocupación del territorio nacional se ha caracterizado por una concentración poblacional progresiva en el arco Andino-Costero del país $\mathrm{y}$, especialmente, en las grandes ciudades ubicadas en el área Centro-Norte, la cual tiene la menor disponibilidad de agua (Rodríguez-Betancourt y González-Aguirre, 2000; Cañizales et al., 2006 citado por Jiménez y Galizia, 2012).

Para satisfacer la demanda de agua en Venezuela se han construido 110 embalses, los cuales tienen usos diversos: suministro de agua potable y para fines industriales, riego, control de inundaciones, recreación y generación de hidroelectricidad, tal y como lo establece el Ministerio del Poder Popular para el Ambiente (MINAMB, 2006). El agua con fines de uso doméstico debe poseer cualidades fisicoquímicas, organolépticas y microbiológicas que reduzcan la probabilidad de ocasionar enfermedades, es decir, se requiere que sea potable.

En Venezuela existen muchas normas regulatorias y leyes relacionadas con el manejo y la conservación de los recursos hídricos, las cuales constituyen en sí mismas ejemplos de buenas prácticas relacionadas con el manejo de dichos recursos. La principal de ellas es la Constitución Nacional de la República Bolivariana de Venezuela (Asamblea Constituyente de Venezuela, 1999). Además de las resoluciones tradicionales de la soberanía nacional sobre sus espacios acuáticos (lacustre y fluvial, mar territorial y aguas marinas interiores). La Constitución establece artículos relacionados con los aspectos ambientales de los recursos hídricos y de la calidad de las aguas, y declara todas las aguas del dominio público del Estado, de conformidad con el Código Civil (MINAMB, 2006).

Asimismo, lo atinente al establecimiento de los valores máximos de aquellos componentes o características del agua que representan un riesgo para la salud de la comunidad, o inconvenientes para la preservación de los sistemas de almacenamiento y distribución del líquido, así como la regulación que asegure su cumplimiento; está estipulado en las Normas Sanitarias de Calidad del Agua Potable (Gaceta Oficial de la República de Venezuela, 1998). A pesar de que existen estas normativas, en Venezuela se han reportado estudios que demuestran deficiencias en el proceso de potabilización, por ejemplo, la presencia de protozoarios intestinales en agua de consumo humano (Guillén et al., 2013) y residuos de plaguicidas (FloresGarcía Molina-Morales, Balza-Quintero, Benítez-Díaz y Miranda-Contreras, 2011).

Uno de los estados de mayor extensión geográfica de Venezuela es el estado Zulia. Esta entidad federal se encuentra situada en el extremo noroccidental del país. Su territorio rodea el lago de Maracaibo, el cual es la masa de agua más extensa de América Latina. La cuenca lacustre abarca una de las más grandes reservas de petróleo y gas del continente americano. Un largo límite fronterizo separa a Venezuela de Colombia por el oeste, desde la Península Guajira hasta 
las montañas de Perijá. Los estados andinos venezolanos Táchira, Mérida y Trujillo limitan con Zulia en la zona sur del lago de Maracaibo y finalmente con los estados Lara y Falcón, completan los límites con el estado Zulia. Éste es un territorio que ha traído enormes riquezas de petróleo al país, pero también es una de las zonas agrícolas importantes de Venezuela, destacando su contribución en áreas como ganadería y agricultura, entre otros. El Zulia es uno de los estados con mayor población (4.120.934 habitantes) y cuenta con una superficie de $63.100 \mathrm{~km}^{2}$ (INE, 2014). Esto a su vez indica que la demanda de agua con calidad es considerable para llevar a cabo las actividades industriales, agrícolas y domésticas.

En el estado Zulia, específicamente el municipio Lagunillas, en la Costa Oriental del Lago de Maracaibo, tiene una superficie de $975 \mathrm{~km}^{2}$ y una población de 239.284 habitantes (INE, 2014). Su capital es Ciudad Ojeda. De relieve plano, se caracteriza por tener un paisaje de bosque seco. La continuada explotación del petróleo ha dado origen al fenómeno de subsidencia del terreno en muchas zonas del municipio, especialmente junto al Lago de Maracaibo, por lo que se ha hecho necesaria la construcción de un dique en la orilla del lago, con la finalidad de impedir la inundación por el desbordamiento del mismo.

Además de contar con riquezas minerales como el petróleo, en el municipio Lagunillas existen infraestructuras para los recursos hídricos de importancia, estos son; una planta de potabilización y un embalse. La planta de Potabilización "Pueblo Viejo" se encuentra en el municipio Lagunillas, en la carretera Lara-Zulia, sus fuentes de abastecimiento son Río Chiquito y Río Grande. Fue construida en el año 1959 y tiene una capacidad de operación de 3.200 litros por segundo. El embalse de Burro Negro tiene una capacidad de 76 mil millones de metros cúbicos de agua y posee una superficie de 1000 hectáreas. Sus fuentes de abastecimiento son Río Chiquito y Río Grande (HIDROLAGO, n.d.).

La parroquia Alonso de Ojeda es una de las que conforman el municipio Lagunillas en el estado Zulia, cuenta con una superficie de $90,30 \mathrm{~km}^{2}$, un total de 55 sectores y 85.482 habitantes (INE, 2014), lo que representa un 42,02 \% de la población total del municipio Lagunillas. Específicamente, en esta parroquia existe la matriz de opinión generalizada entre sus habitantes de que el agua recibida en sus hogares no reúne las características requeridas en cuanto a potabilidad, negándose a consumir el agua que llega directamente a sus domicilios, prefiriendo surtirse de este recurso en el comercio privado y comprar filtros purificadores costosos e instalarlos en sus viviendas, entre otras medidas.

La situación descrita implica que probablemente el agua que se suministra por la red de distribución no presenta características fisicoquímicas y microbiológicas que cumplan con las normas vigentes, a pesar de contar con una infraestructura adecuada para ello y que un estudio previo realizado en el casco central de Ciudad Ojeda arrojó valores de turbidez, color, $\mathrm{pH}$ y alcalinidad ajustados a la normativa vigente, así como ausencia de coliformes fecales y de la bacteria Escherichia coli (Salas, 2011).

En función de lo expuesto, se consideró interesante determinar la calidad del agua de uso doméstico en la parroquia Alonso de Ojeda, municipio Lagunillas del estado ZuliaVenezuela. 


\section{MATERIALES Y MÉTODOS}

\section{Período y localización del muestreo}

La investigación se ubicó en el nivel descriptivo y se empleó un diseño de campo. La población de estudio estuvo representada por el agua que llega a los hogares a través de la red de distribución que abastece la parroquia Alonso de Ojeda, municipio Lagunillas del estado Zulia (Venezuela).
La muestra estuvo representada por el agua de grifo de 100 viviendas pertenecientes a la mencionada parroquia. En un período de cuatro meses (mayoagosto de 2016) se recolectaron al azar muestras de agua en diferentes puntos de la parroquia Alonso de Ojeda, municipio Lagunillas del estado Zulia-Venezuela (Figura 1).

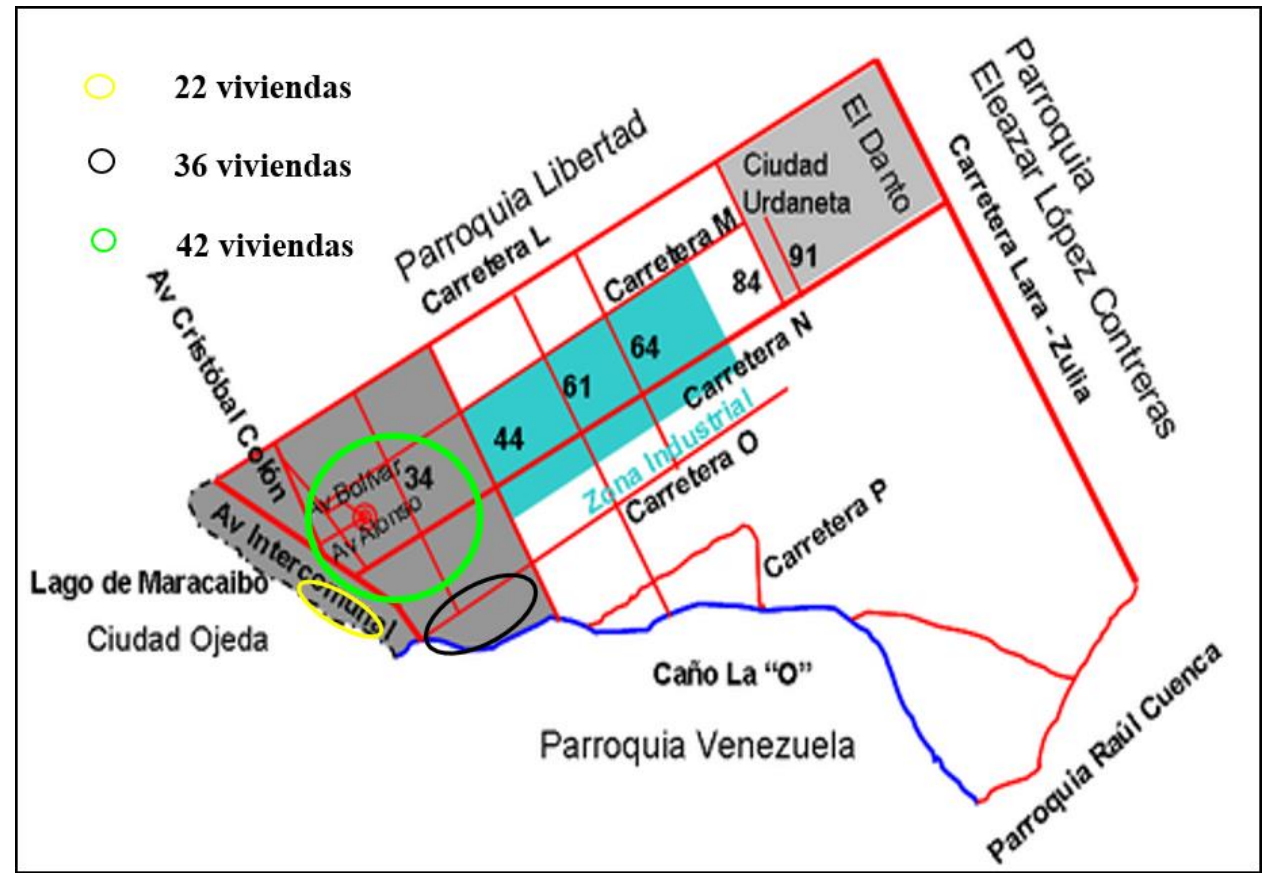

Figura 1. Áreas de recolección de muestras en la parroquia Alonso de Ojeda. Fuente: Strauss et al. (2000).

\section{Procedimiento para la recolección de muestras}

Se utilizaron recipientes de vidrio esterilizados con capacidad de $1000 \mathrm{~mL}$ para la recolección de muestras y se trasladaron al laboratorio conservándolos a temperaturas entre 4 y $10{ }^{\circ} \mathrm{C}$. La toma de muestras se realizó en cada grifo de una cañería de agua corriente, eligiendo un grifo conectado directamente con una cañería de distribución, es decir, la que conduce a la cañería principal.
Se quitó del grifo cualquier accesorio, boquilla de goma u otro, y donde se observó la presencia de sedimentos acumulados, se retiraron. Con las manos esterilizadas con alcohol se drenó el agua del grifo por 5 minutos y se cerró la salida del agua. Se desinfectó con alcohol y se flameó con un mechero el punto de toma, para finalmente dejar fluir el agua por 2-3 minutos en forma controlada evitando salpicaduras, ubicando el recipiente debajo del grifo dejando que se 
llenara a $3 / 4$ de su capacidad y verificando que quedara una capa de aire en el cuello del envase, cerrándose rápidamente para evitar su contaminación (Norma Venezolana COVENIN 2614-94, 1994).

\section{Medición de parámetros \\ fisicoquímicos}

La técnica que se utilizó para esta investigación fue la observación asistida
(Hurtado, 2010) por medio de diferentes equipos y materiales. Se determinaron los parámetros turbidez, color, $\mathrm{pH}$ y alcalinidad mediante el empleo de los métodos y procedimientos recomendados en el Standard Methods (APHA-AWWA-WEF, 1998), tal y como se describen en la Tabla 1.

Tabla 1. Equipos, materiales y métodos para la determinación de parámetros fisicoquímicos.

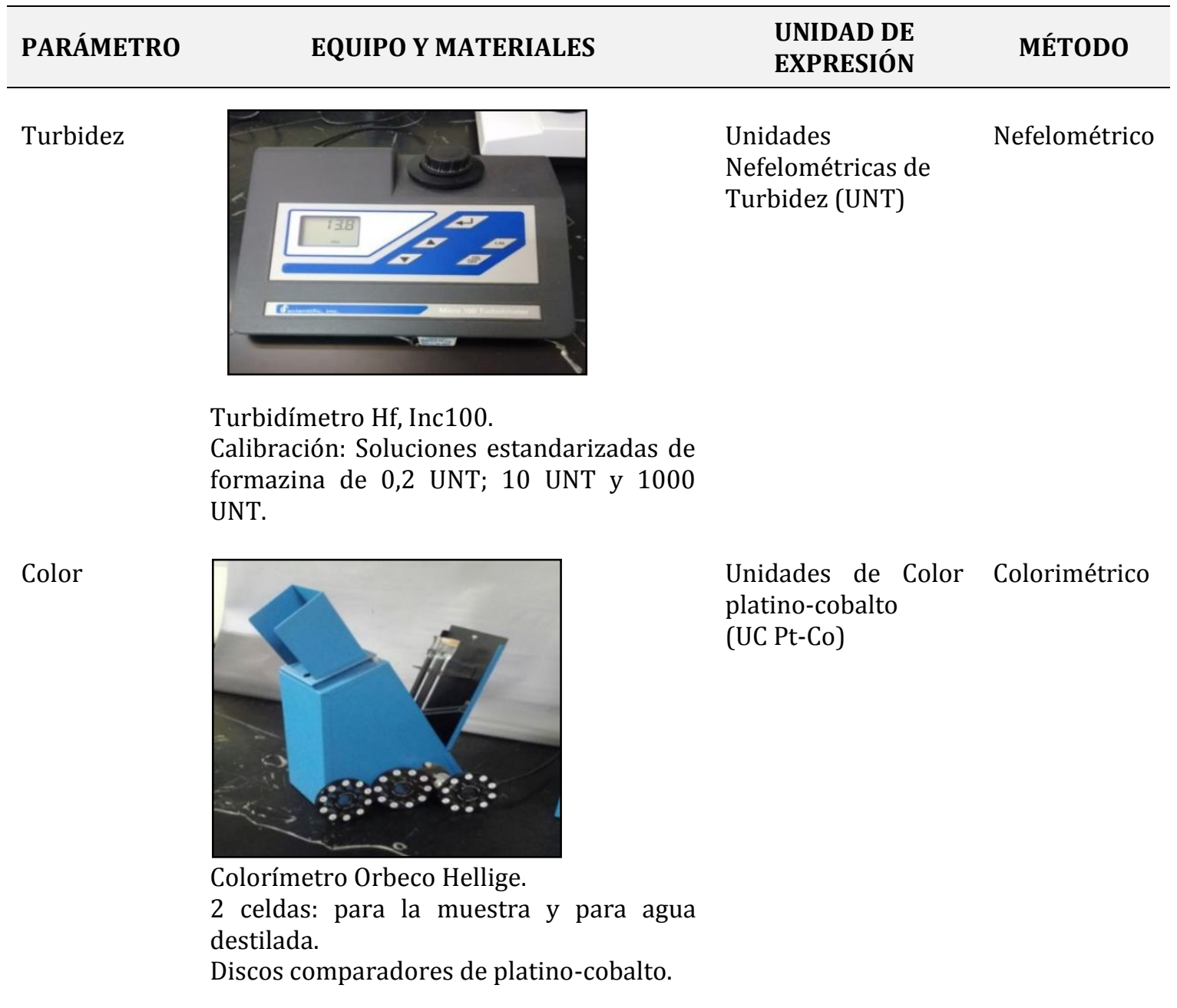




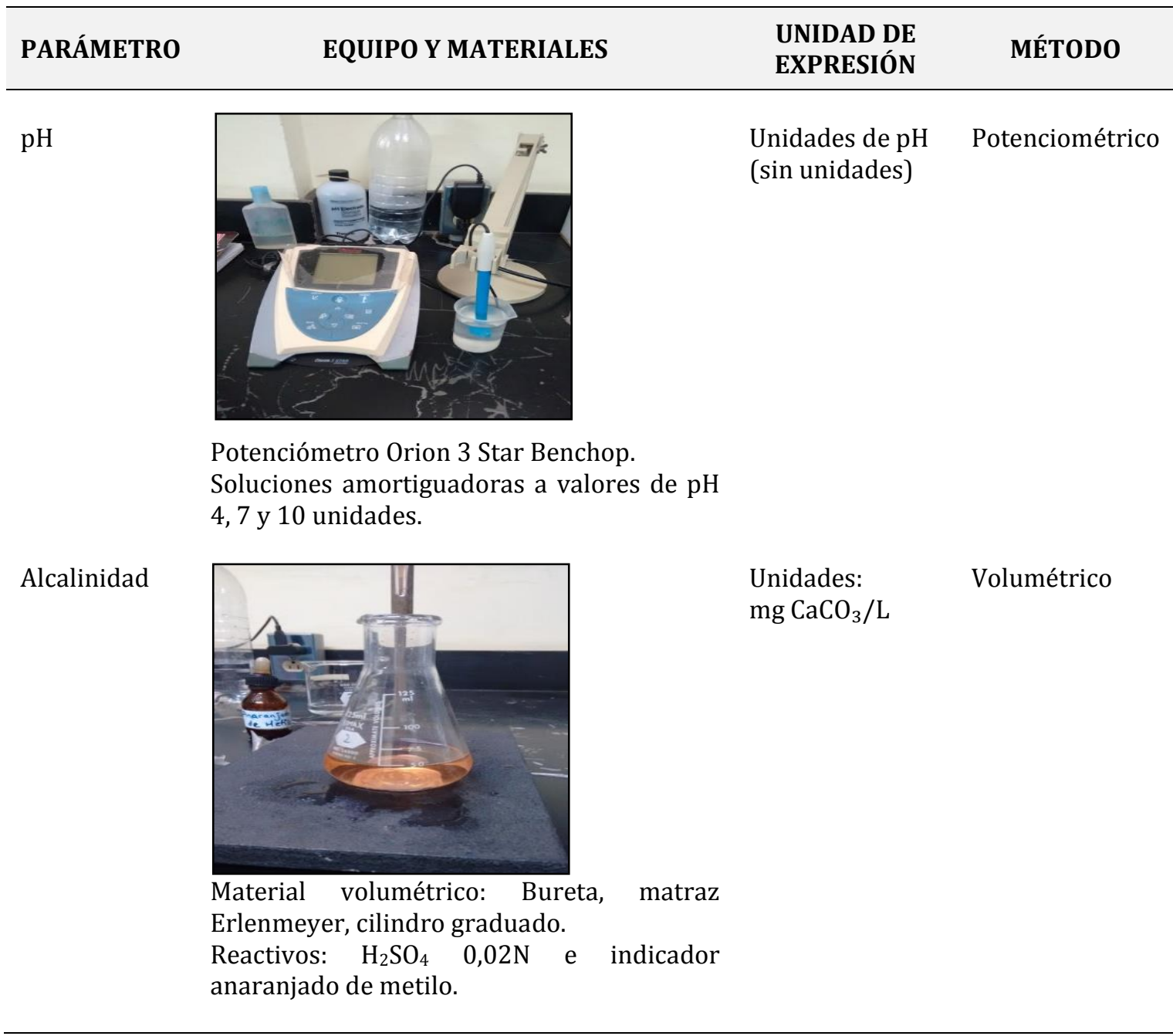

\section{Medición de parámetros} microbiológicos

Se aplicó el Método del Número Más Probable (NMP), según la norma venezolana COVENIN 1104 (1996) para la determinación de coliformes totales mediante las pruebas presuntiva $\mathrm{y}$ confirmatoria. La positividad de las muestras se estableció a partir de la observación de turbidez o gas en los tubos inoculados. Se emplearon cinco tubos de muestra y después de contar el número de tubos obtenidos, el resultado se comparó con lo estipulado en las tablas de Número Más Probable de la norma venezolana COVENIN 3047 (1993).
Los resultados se expresaron como NMP/100 mL de muestra y su interpretación se hace de la siguiente manera: si ningún tubo resulta positivo, el resultado se expresa como $\leq 2,2 \mathrm{NMP} / 100 \mathrm{~mL}$, si se observa un (1) tubo positivo el resultado se expresa como 2,2 NMP /100 mL muestra, cuando son dos (2) tubos (5,1 NMP /100 mL), tres (3) tubos (9,2 NMP /100 mL), cuatro (4) tubos (16 NMP /100 mL) y en caso de que todos los tubos sean positivos, el resultado se expresa como $\leq 16,0 \mathrm{NMP} / 100 \mathrm{~mL}$ muestra. Adicionalmente, se aplicaron las pruebas presuntiva y confirmatoria a muestras de agua destilada para utilizarlas como control en el estudio (Figura 2). 
Autoclave: Esterilización de instrumentos y medios de cultivo.

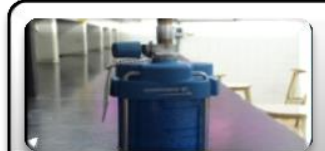

Mechero: Acondicionamiento del ambiente, desinfección de asa de inoculación y boca de los tubos de ensayo.

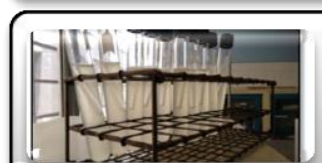

Prueba presuntiva: Adición de muestras de agua a tubos de ensayo con caldo lactosado e incubación en la estufa.

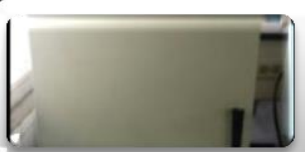

Estufa: Para la incubación de las muestras en las fases presuntiva y confirmatoria.
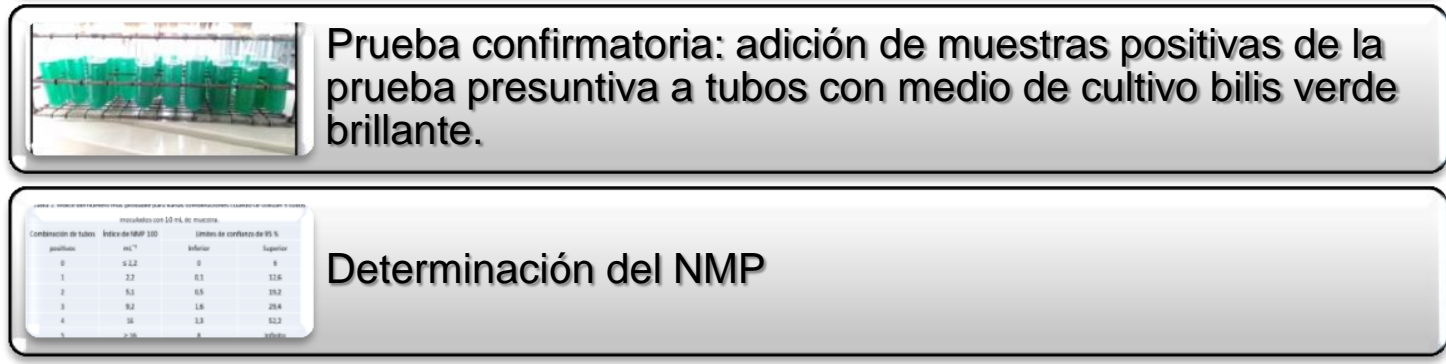

Figura 2. Procedimiento para la determinación de coliformes totales mediante el NMP

\section{RESULTADOS Y DISCUSIÓN}

Calidad fisicoquímica de las aguas destinadas al consumo humano en la parroquia Alonso de Ojeda

Se presenta a continuación un análisis de los valores de turbidez, color, $\mathrm{pH}$ y alcalinidad obtenidos en cien (100) muestras de agua destinada al consumo humano en la parroquia Alonso de Ojeda, municipio Lagunillas del estado ZuliaVenezuela.

\section{Turbidez}

El parámetro turbidez del agua de uso doméstico en la parroquia Alonso de Ojeda osciló entre 1,68 UNT y 92 UNT, valores mínimo y máximo, respectivamente (Figura 3). Se indica que este parámetro no cumplió en su totalidad con las normas establecidas, teniendo un promedio de 22,86 UNT. Esto evidencia que el agua presenta una turbiedad fuera de lo permisible, según lo establecido en las Normas Sanitarias de Calidad del Agua Potable de Venezuela (Gaceta Oficial de la República de Venezuela, 1998), ya que ésta no debe superar el valor de 5 UNT. Adicionalmente, se determinó que el $7 \%$ de las muestras cumplen con la normativa establecida, pero el $93 \%$ no se ajusta a la misma. 


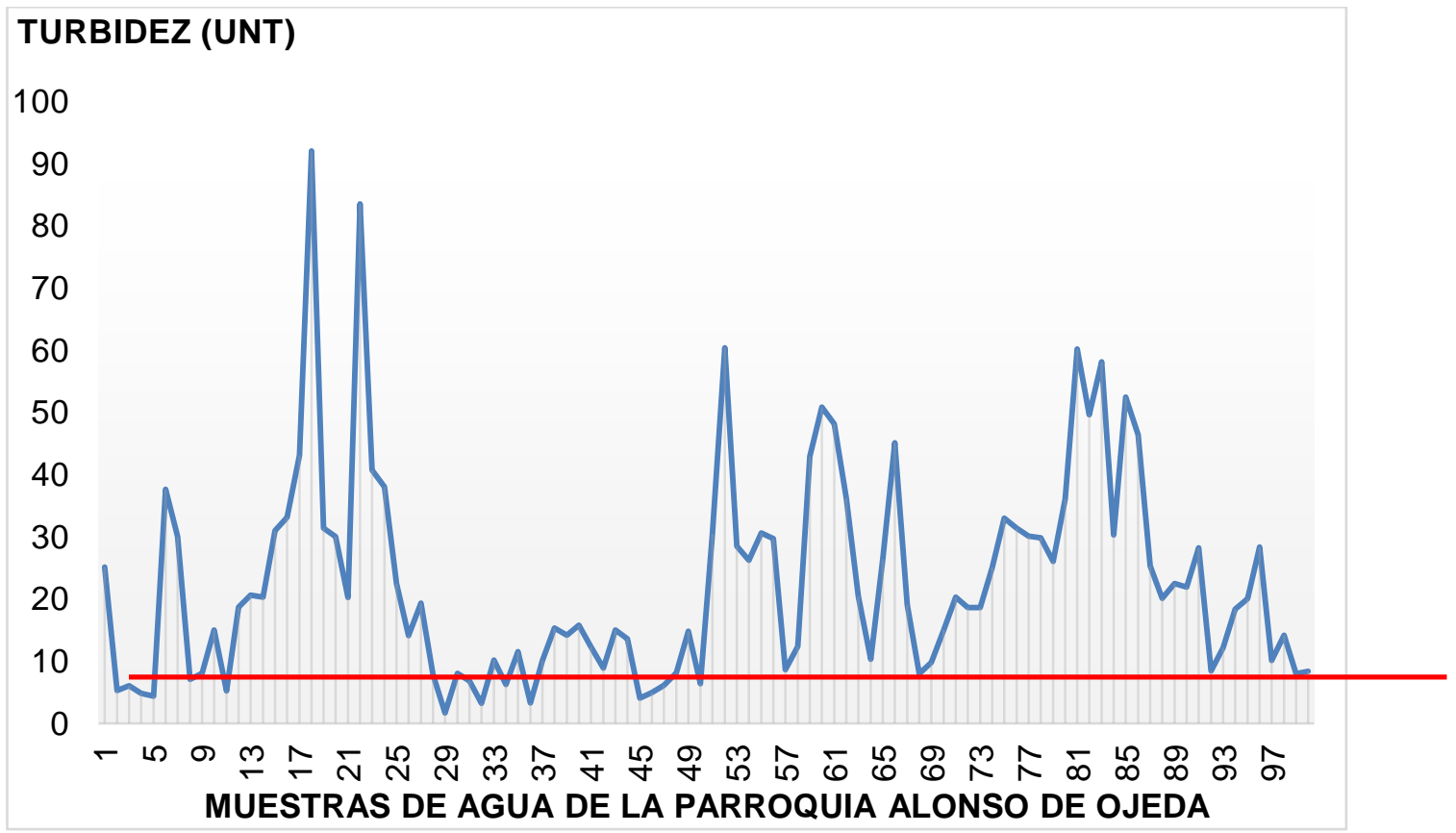

Figura 3. Dispersión lineal con valores de turbidez en muestras de agua destinada al consumo humano en la parroquia Alonso de Ojeda.

Los resultados fueron superiores a los reportados por Mejía (2005), quien indicó un valor promedio de 7 UNT al evaluar el agua de la microcuenca El Limón en Honduras. También difirieron de los señalados por Pérez (2010), quien caracterizó el agua potable distribuida por una red de abastecimiento en la ciudad de Yopal (Colombia) y reportó un valor de 3 UNT; de los reflejados por Fajardo et al (2017) al estudiar la calidad del agua en un barrio de Bogotá, determinando un promedio de 0,32 UNT; y de los obtenidos por Salas (2011) al estudiar la calidad del agua distribuida en el casco central de Ciudad Ojeda, municipio Lagunillas, quien detectó valores de turbidez que fluctuaron entre 2,83 y 5,09 UNT con un promedio de turbidez de 3,86 UNT, lo cual refleja que para ese año el agua cumplía con las normas establecidas en la zona investigada.

\section{Color}

Los resultados de las muestras no se ajustan a las normas establecidas que indican un intervalo entre $0 \mathrm{UC}$ Pt-Co a 15 UC Pt-Co (Gaceta Oficial de la República de Venezuela, 1998). Los valores obtenidos para el parámetro color fluctuaron entre 20 UC Pt-Co y 200 UC Pt-Co, evidenciándose el incumplimiento de las referidas normas. El promedio de este parámetro se ubicó en 39,6 UC Pt-Co, por lo cual, desde un punto de vista general, el agua destinada al consumo humano en la parroquia Alonso de Ojeda no cumple con lo referido para el color en las normas para la calidad del agua vigentes de Venezuela (Figura 4).

Comparando estos resultados con los obtenidos por Salas (2011), se observan diferencias considerables, pues las muestras captadas por esta investigadora arrojaron un resultado aceptable de 5 UC Pt-Co al igual que Fajardo et al. (2017) al registrar 0 UPC 
(Unidades de platino-cobalto). Asimismo, los valores de color del presente estudio difieren de los reportados por Pérez (2010), pero son similares a los indicados por Mejía
(2005), quienes en sus investigaciones reflejaron valores de $10 \mathrm{UC}$ Pt-Co y $61 \mathrm{UC}$ PtCo, respectivamente.

\section{COLOR (UC Pt-Co) \\ 250 \\ 200 \\ 100 \\ 50 \\ IV \\ 0

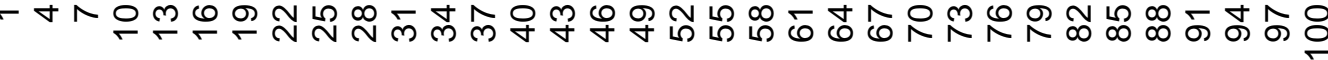 \\ MUESTRAS DE AGUA DE LA PARROQUIA ALONSO DE OJEDA}

Figura 4. Dispersión lineal con valores de color en muestras de agua destinada al consumo humano en la parroquia Alonso de Ojeda.

ph

En la Figura 5 se observa un comportamiento en el índice de $\mathrm{pH}$ variante entre 6,15 y 7,40 unidades, siendo estos los valores mínimo y máximo, respectivamente. Desde el punto de vista general se obtuvo un promedio de 6,55 unidades, situándose en el rango de 6,5-8,5, aceptado conforme a las Normas Sanitarias de Calidad del Agua Potable de Venezuela (Gaceta Oficial de la República de Venezuela, 1998). Igualmente se refleja que un $38 \%$ de las muestras estuvieron por debajo de 6,5 y el $62 \%$ de las muestras estuvo dentro de lo aceptado por las normas venezolanas.
Los resultados en cuanto al parámetro pH son similares a los expuestos por Pérez (2010), quien reportó un promedio de 6,85 unidades. Adicionalmente, al contrastar con Salas (2011), se evidencia que también obtuvo valores en un rango permisible para la misma zona de estudio, los cuales variaron desde 7,31 hasta 7,85 unidades, cumpliendo en un $100 \%$ con las Normas Sanitarias de Calidad del Agua Potable de Venezuela (Gaceta Oficial de la República de Venezuela, 1998). 


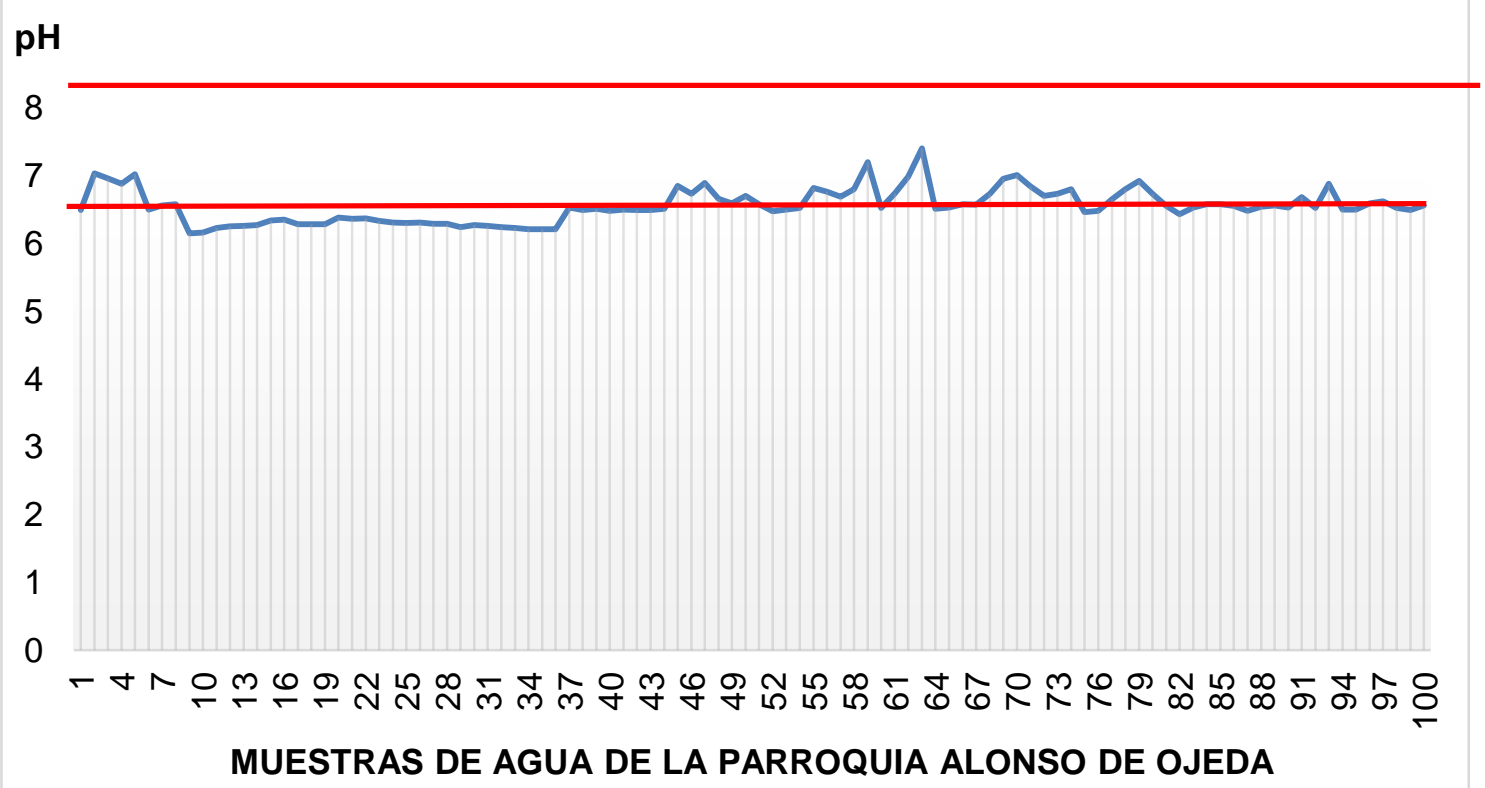

Figura 5. Dispersión lineal con valores del potencial de hidrógeno $(\mathrm{pH})$ en muestras de agua destinada al consumo humano en la parroquia Alonso de Ojeda.

\section{Alcalinidad}

Si bien es conocido que en las normas venezolanas de calidad del agua potable no hay una estándar que fije límites para la alcalinidad en el agua, existen otros países que dentro de sus normas han establecido límites para la misma, siendo el caso del país vecino (Colombia) en su resolución 2115 del Ministerio de la Protección Social, Ministerio de Ambiente, Vivienda y Desarrollo Territorial de Colombia (2007), la cual establece un valor máximo aceptable de $200 \mathrm{mg} \mathrm{CaCO}_{3} / \mathrm{L}$. De conformidad con este estándar, las muestras obtenidas se ubican por debajo del máximo aceptable y se concluye que cumplen con el mismo. Los valores de alcalinidad oscilaron desde 6,00 $\mathrm{mg} \mathrm{CaCO}_{3} / \mathrm{L}$ hasta $52,0 \mathrm{mg} \mathrm{CaCO}_{3} / \mathrm{L}$ y se obtuvo un promedio de $14,98 \mathrm{mg} \mathrm{CaCO}_{3} / \mathrm{L}$ (Figura 6), cercano al obtenido por Fajardo et al. (2017), cuyo promedio fue de 18,10 $\mathrm{mg} \mathrm{CaCO} / \mathrm{L}$. 


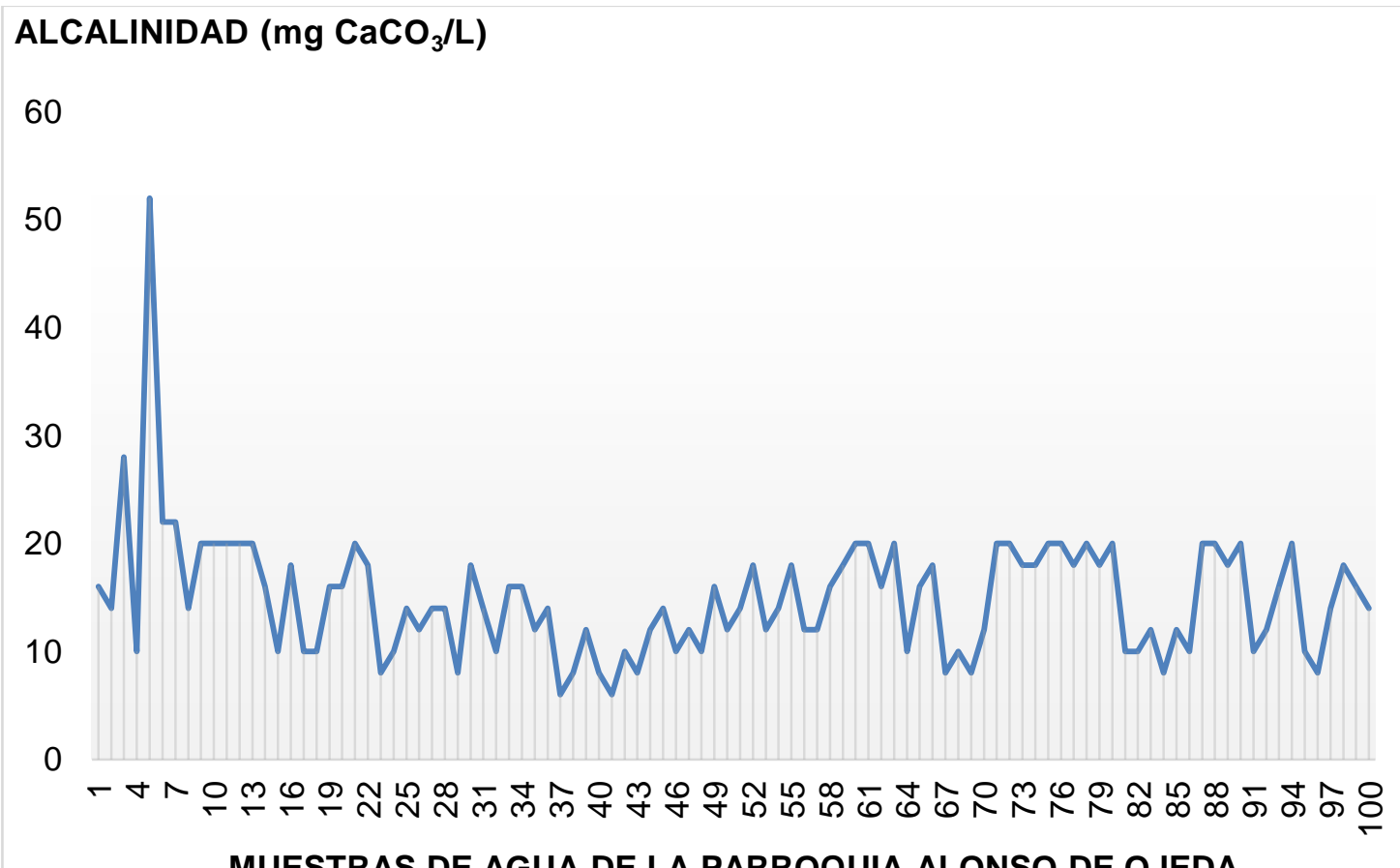

Figura 6. Dispersión lineal con valores de alcalinidad $\left(\mathrm{mg} \mathrm{CaCO}_{3} / \mathrm{L}\right)$ en muestras de agua destinada al consumo humano en la parroquia Alonso de Ojeda.

En comparación con Gramajo (2004), quien reportó un valor promedio de $186 \mathrm{mg}$ $\mathrm{CaCO}_{3} / \mathrm{L}$, los valores de alcalinidad de la presente investigación resultaron inferiores; y al contrastar con los referidos por Salas (2011), quien indica valores de 40-70 mg $\mathrm{CaCO}_{3} / \mathrm{L}$, y por Estupiñán y Ávila (2010) al obtener un rango entre 90-140 $\mathrm{mg} \mathrm{CaCO}_{3} / \mathrm{L}$, se observa también que la alcalinidad se ubica en niveles inferiores, pero en todos los casos se ajustan a la normativa vigente en Colombia.

En cuanto a los parámetros fisicoquímicos evaluados (turbidez, color, $\mathrm{pH}$ y alcalinidad), el agua que se suministra por la red de distribución a la parroquia Alonso de Ojeda no es apta para el consumo humano, debido a que la turbidez y el color no cumplen con los valores permisibles establecidos en las normas vigentes.

Calidad microbiológica de las aguas destinadas al consumo humano en la parroquia Alonso de Ojeda

De las cien muestras se realizaron 25 corridas, es decir, un análisis microbiológico cada cuatro muestras fisicoquímicas, cabe destacar que a estas muestras se les caracterizó fisicoquímicamente antes de realizar las pruebas microbiológicas. 


\section{Prueba presuntiva}

En la prueba presuntiva se observó claramente la presencia de gas dentro de los tubos Durham, y fue tanta la cantidad de gas formado, que en cada corrida se observó de forma frecuente que los tubos Durham se elevaban del fondo del tubo de ensayo quedando en suspensión (Figura 7). Paras las 25 corridas en todos los tubos de ensayo (5 tubos con muestra del agua de grifo y caldo lactosado para cada corrida) se observó formación de gas y turbiedad, es decir, resultaron positivas.

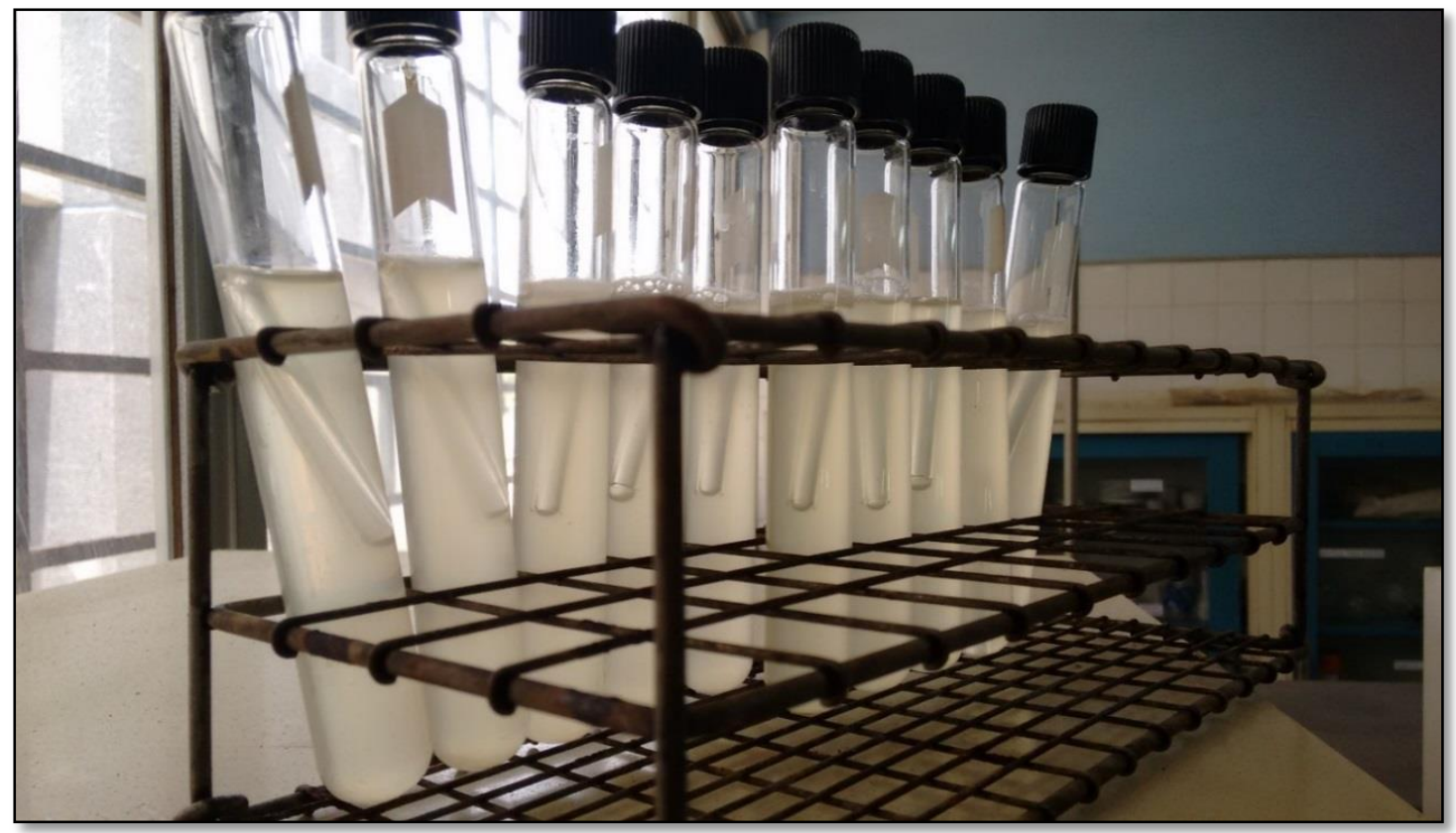

Figura 7. Muestras del resultado de la prueba presuntiva.

\section{Prueba confirmatoria}

En la prueba confirmatoria los resultados fueron positivos, no estuvieron alejados de los obtenidos en la prueba presuntiva, solo en una corrida no se observó la presencia de gas dentro de los cinco tubos Durham. Para 24 muestras de agua destinada al consumo humano los cinco tubos resultaron positivos y arrojaron un valor $\geq 16 \mathrm{NMP} / 100 \mathrm{~mL}$. En una muestra se obtuvo un valor de $16 \mathrm{NMP} / 100 \mathrm{~mL}$, ya que solo 4 tubos resultaron positivos (Figura 8). 


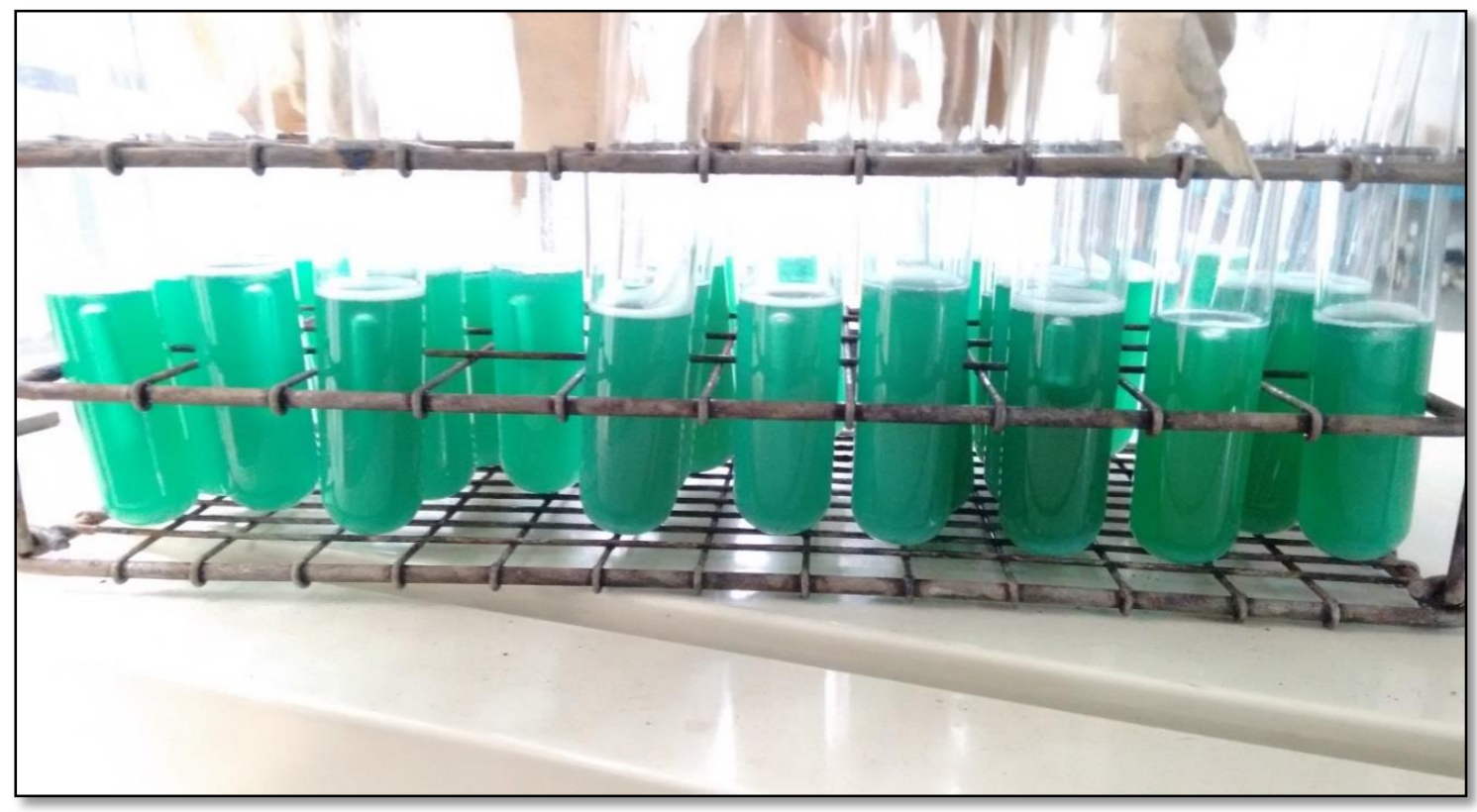

Figura 8. Muestras del resultado de la prueba confirmatoria.

Los resultados indican el $100 \%$ de contaminación por coliformes totales en las muestras de agua evaluadas en la parroquia Alonso de Ojeda del municipio Lagunillas del estado Zulia (Venezuela), discriminados de la siguiente manera: $4 \%$ de las muestras con
$16 \mathrm{NMP} / 100 \mathrm{~mL}$ y $96 \%$ de las muestras $\geq$ $16 \mathrm{NMP} / 100 \mathrm{~mL}$. Adicionalmente, se observó que las corridas con agua destilada como muestras de control, arrojaron valores de coliformes totales $=0 \mathrm{NMP} / 100 \mathrm{~mL}$ (Tabla 2).

Tabla 2. Índice del Número Más Probable (NMP) para aguas de uso doméstico y destilada.

\begin{tabular}{llllll}
\hline Parámetro & Referencia & \multicolumn{2}{l}{ Agua de uso doméstico } & \multicolumn{2}{l}{ Agua destilada } \\
& & Frecuencia & $\mathbf{\%}$ & Frecuencia & $\mathbf{\%}$ \\
\cline { 3 - 6 } & $\leq 2,2$ & 0 & 0 & 2 & 100 \\
Coliformes totales & 16 & 1 & 4 & 0 & 0 \\
(NMP/100 mL) & $\geq 16$ & 24 & 96 & 0 & 0 \\
\hline
\end{tabular}

Los resultados contrastan con el valor de $1 \mathrm{NMP} / 100 \mathrm{~mL}$ obtenido por Salas (2011) para coliformes totales, quien además determinó que no había rastro de coliformes fecales, lo cual resulta de mucho interés tomando en cuenta que ambas investigaciones coinciden en cuanto a la zona geográfica de estudio (Ciudad Ojeda), con una diferencia de tiempo correspondiente a 5 años. Asimismo, Baque-
Mite et al. (2016) refirieron valores de 1,0 NMP/100 mL para coliformes totales y para coliformes fecales.

Por otra parte, los resultados del presente estudio son similares a los reportados por Bríñez et al. (2012) al indicar un valor de 15,0 NMP/100 mL para coliformes totales, y por Mejía (2005), cuyo trabajo mostró valores de coliformes totales $=14,54 \mathrm{NMP} / 100 \mathrm{~mL}$ y coliformes fecales $=$ 
$960 \mathrm{NMP} / 100 \mathrm{~mL}$, resultando no apta para el consumo humano.

La presencia de coliformes totales en las muestras de agua de uso doméstico analizadas, permite inferir que el agua en la parroquia "Alonso de Ojeda" no es apta para el consumo humano, pues contraviene lo establecido en la normativa venezolana, conforme a la cual, en ningún caso deberá detectarse organismos coliformes totales en dos muestras consecutivas de $100 \mathrm{~mL}$, provenientes del mismo sitio (Gaceta Oficial de la República de Venezuela, 1998).

Estos resultados pueden incidir en problemas de salud de la población, principalmente gastrointestinales, constituyendo un riesgo, lo cual coincide con lo aseverado por Brínez et al. (2012), pues una inadecuada calidad microbiológica del agua, sumado a algún tipo de riesgo en el agua para consumo humano, constituyen uno de los potenciales más peligrosos para la salud humana al ser un líquido de uso permanente y necesario.

En síntesis, el agua de uso doméstico distribuida en la parroquia Alonso de Ojeda del municipio Lagunillas del estado Zulia está contaminada, pudiendo atribuirse estos resultados a fallas en el tratamiento del agua o problemas de abastecimiento, es decir, ausencia del agua en el sistema de suministro por períodos mayores a 24 horas, y es que en esta zona hay lapsos hasta de 120 horas (5 días) sin la presencia del vital líquido en la red de distribución; de modo que al regresar el flujo del agua en el sistema, arrastra todas las partículas y sedimentos allí presentes.

\section{CONCLUSIONES}

La evaluación de la calidad fisicoquímica del agua de uso doméstico en la parroquia Alonso de Ojeda, municipio
Lagunillas del estado Zulia reveló que los valores promedio de los parámetros fisicoquímicos evaluados (pH: 6,55, turbidez: 22,86 UNT, color: 39,6 UC Pt-Co y alcalinidad: 14,98 $\mathrm{mg} \mathrm{CaCO}_{3} / \mathrm{L}$ ), a excepción del $\mathrm{pH}$ y la alcalinidad, están fuera de los niveles permisibles, es decir, la turbidez y el color no cumplen con las normas de calidad de agua potable vigentes en Venezuela. En cuanto al parámetro alcalinidad se refiere, éste se comparó con estándares colombianos vigentes de calidad del agua potable para consumo humano, ubicándose en promedio dentro de los parámetros deseados, siendo el límite permisible por las normas colombianas de $200 \mathrm{mg}$ $\mathrm{CaCO}_{3} / \mathrm{L}$.

La determinación de la calidad microbiológica mediante el Número Más Probable demostró la presencia de coliformes totales ( $4 \%$ de las muestras con $16 \mathrm{NMP} / 100 \mathrm{~mL}$ y $96 \%$ de las muestras $\geq$ $16 \mathrm{NMP} / 100 \mathrm{~mL}$ ), lo cual contraviene lo establecido en la normativa venezolana, resultando no apta para el consumo humano.

\section{REFERENCIAS}

Asamblea Constituyente de Venezuela (1999). Constitución de la República Bolivariana de Venezuela. Gaceta Oficial $\mathrm{N}^{\circ}$ 5.453. Marzo 24, 2000

APHA, AWWA, WEF (1998). Standard methods for the examination of water and wastewater (20th ed.). American Public Health Association/American Water Works Association/Water Environmental Federation. 20th Washington, DC, USA

Baque-Mite, R., Simba-Ochoa, L., GonzálezOzorio, B., Suatunce, P., Díaz-Ocampo, E., Cadme-Arevalo, L. (2016). Calidad del agua destinada al consumo humano en un cantón de Ecuador. Revista Ciencia UNEMI, 9(20), 109-117. DOI: 
https://doi.org/10.29076/issn.25287737vol9iss20.2016pp109-117p

Bríñez, K., Guarnizo, J. y Arias, S. (2012). Calidad del agua para consumo humano en el departamento del Tolima. Rev. Fac. Nac. Salud Pública, 30(2), 175-182

Estupiñán, S. y Ávila, S. (2010). Calidad fisicoquímica y microbiológica del agua del municipio de Bojacá, Cundinamarca (Colombia). NOVA-Publicación Científica en Ciencias Biomédicas, 8(14), 121-240

Fajardo, Á., Gaines, S., Muñoz-Silva, V., Otero, V. y Mendoza, V. (2017). Calidad del agua y características habitacionales de un barrio en Bogotá. Nova, 15(27), 31-36.

DOI: https://doi.org/10.22490/24629448.1 956

Flores-García, M., Molina-Morales, Y., BalzaQuintero, A., Benítez-Díaz, P., MirandaContreras, L. (2011). Residuos de plaguicidas en aguas para consumo humano en una comunidad agrícola del estado Mérida, Venezuela. Investigación Clínica, 52(4), 295-311

Gaceta Oficial de la República de Venezuela (1998). Normas Sanitarias de Calidad del Agua Potable. № 36.395 del 13 de febrero de 1998. Ministerio de Sanidad y Asistencia Social. Caracas, Venezuela. p.p. 8

Gramajo, M. (2004). Determinación del agua para consumo humano y uso industrial, obtenida de pozos mecánicos en la zona 11, Mixco, Guatemala [Trabajo de Graduación, Universidad de San Carlos de Guatemala]. San Carlos de Guatemala, Guatemala

Guillén, A., González, M., Gallego, L., Suárez, B., Heredia, H., Hernández, T., Naranjo, M. y Salazar, J. (2013). Presencia de protozoarios intestinales en agua de consumo en la comunidad 18 de Mayo. Estado Aragua-Venezuela, 2011. Boletín de Malariología y Salud Ambiental, 53(1), 29-36

HIDROLAGO (n.d.). Hidrocuencas. Recuperado http://www.hidrolago.gov.ve/hidrocue ncas.html

Hurtado, J. (2010). Metodología de la investigación. Tercera Edición. Caracas: Fundación Sypal

Instituto Nacional de Estadística. INE. (2014). Censos. Recuperado de http://www.ine.gov.ve/documentos/D emografia/CensodePoblacionyVivienda /pdf/zulia.pdf

Jiménez, B. y Galizia, J. (2012). Diagnóstico del agua en las Américas. Foro Consultivo, Científico y Tecnológico (447 p.p.). AC. Interamerican Network of Academies o Sciences

Mejía, M. (2005). Análisis de la calidad del agua para consumo humano $y$ percepción local de las tecnologías apropiadas para su desinfección a escala domiciliaria, en la microcuenca El Limón, San Jerónimo, Honduras. [Tesis de Postgrado, Centro Agronómico Tropical de investigación y Enseñanza]. Turrialba, Costa Rica

Ministerio de la Protección Social, Ministerio de Ambiente, Vivienda y Desarrollo Territorial de Colombia (2007). Resolución Numero 2115. Bogotá, Colombia. 23 p.p.

Ministerio del Poder Popular para el Ambiente. MINAMB. (2006). Recursos hídricos de Venezuela. Ministerio del Ambiente y Fundambiente. Caracas. 167 p.

Norma Venezolana Agua Potable. COVENIN 3047-93 (1993). Determinación de NMP. Tabla 1 y 2. Anexos II. Caracas

Norma Venezolana COVENIN 2614-94 (1994). Agua potable. Toma de muestra. Primera revisión. Caracas

Norma Venezolana COVENIN 1104-96 (1996). Determinación del número más probable de coliformes, coliformes fecales y de Escherichia coli. Caracas

Organización de las Naciones Unidas para la Educación, la Ciencia y la Cultura. UNESCO. (2011). Estadística de la tasa de mortalidad a consecuencia de aguas 
contaminadas y contaminación del agua a consecuencia del uso humano

Pérez, J. (2010). Caracterización de la calidad del agua en la planta de tratamiento de agua potable y en la red de distribución de la ciudad de Yopal. [Trabajo Especial de Grado, Universidad Industrial de Santander]. Bucaramanga, Colombia

Programa Ambiental de las Naciones Unidas. UNEP. (2010). Porcentaje de agua dulce en el planeta tierra

Rodríguez-Betancourt, R. y GonzálezAguirre, J. (2000). El manejo de los recursos hídricos en Venezuela. IWMI, Serie Latinoamericana: No. 18. México, D.F, México: Instituto Internacional del Manejo de Agua

Salas, Y. (2011). Calidad del agua distribuida en el casco central de Ciudad Ojeda, Municipio Lagunillas. [Trabajo Especial de Grado, Universidad del Zulia]. Cabimas, Venezuela

Strauss, E., Fuenmayor, W. y Romero, J. (2000). Atlas del Estado Zulia 